Randomized Control Trial

\title{
Pulsed Radiofrequency Treatment for Chronic Post-Surgical Orchialgia: A Double-Blind, Sham-Controlled, Randomized Trial: Three- Month Results
}

Diab Fuad Hetta, MD¹, Ali Mohammed Mahran, MD², and Emad Eldien Kamal, MD²

From: ${ }^{1}$ South Egypt Cancer Institute, Assuit University,

Egypt; ${ }^{2}$ Assuit University Hospital, Assuit University, Egypt

Dr. Hetta is a lecturer in Anesthesia and Pain Management, South Egypt Cancer Institute, Assuit University, Egypt. Dr.s. Mahran and Kamal are Associate

Professors of Andrology and

Sterility, Assuit University Hospital, Assuit University, Egypt

Address Correspondence: Diab Fuad Hetta, MD

Department of Anesthesia and Pain Management

South Egypt Cancer Institute, Assuit University 71111 El-methaque St.

City District, Assuit City, Egypt E-mail: diabhetta25@gmail.com

Disclaimer: There was no external funding in the preparation of this manuscript. Conflict of interest: Each author certifies that he or she, or a member of his or her immediate

family, has no commercial association (i.e., consultancies, stock ownership, equity interest, patent/licensing arrangements, etc.) that might pose a conflict of interest in connection with the submitted manuscript.

Manuscript received: 04-13-2017 Revised manuscript received: 07-15-2017

Accepted for publication: 09-25-2017

Free full manuscript: www.painphysicianjournal.com
Background: Chronic post-surgical pain in the groin region represents a challenge for the managing physician and is a burden on the quality of life of the patient. None of the existing interventions or medical treatment is satisfactory.

Objectives: We aim to evaluate the analgesic efficacy of pulsed radiofrequency (PRF) applied to the ilioinguinal nerve and the genital branch of the genitofemoral nerve for patients suffering from chronic post-surgical orchialgia.

Study Design: A prospective randomized, controlled clinical trial.

Settings: An interventional pain unit in a tertiary center at a university hospital in Egypt.

Methods: Seventy patients complaining of chronic post-surgical orchialgia were randomized into 2 groups: PRF group $(n=35)$, received pulsed radiofrequency on the ilioinguinal nerve and genital branch of the genitofemoral nerve, or sham group $(n=35)$. The percentage of patients that showed $>50 \%$ reduction of their visual analog scale (VAS) pain score as well as the percentage of patients that did not require additional analgesic drugs was assessed. The VAS pain score and the global perceived effect (GPE) were reported during the 3-month follow-up period.

Results: The percentage of patients that showed $>50 \%$ reduction of their VAS pain score was $80 \%(24 / 30)$ in the PRF group versus $23.33 \%(7 / 30)$ in the sham group. The percentage of patients that did not require analgesic drugs was $50 \%(15 / 30)$ in the PRF group versus $3.3 \%(1 / 30)$ in the sham group. There was a significant reduction of the mean post-procedural VAS pain score at 2, $4,6,8$, and 12 weeks $(P=0.001)$ in the PRF group in comparison to the sham group. Likewise, there was a significant improvement of the GPE in the PRF group in comparison to the sham group $(P=0.00)$

Limitations: The study's follow-up period was limited to 3 months only.

Conclusions: For patients suffering from chronic post-surgical orchialgia, PRF applied to the ilioinguinal nerve and the genital branch of the genitofemoral nerve is an effective treatment modality. It provides long-lasting pain relief and decreases the demand for pain medications.

Key words: Orchialgia, groin pain, radiofrequency, ilioinguinal nerve, genitofemoral nerve

Trial Registry: www.anzctr.org.au, ACTRN12616000649415.

Pain Physician 2018; 21:199-205 hronic post-groin surgery orchialgia represents a challenge for the managing physicians and is Ja burden on the quality of life of the patients. It has been reported that the incidence of chronic post-inguinal herniorrhaphy pain of moderate to severe nature resembles $10-12 \%$ (1), and the daily life activities are affected in approximately 0.5-6\% (2). Chronic orchialgia of various etiologies affects up to 
100,000 men in the US yearly. In the UK, the incidence has been estimated to be around $1 \%(3)$.

Chronic post-surgical pain in the groin region is mostly due to injury or entrapment of the ilioinguinal and/or the genitofemoral nerves during surgical dissections (4). Unfortunately, the anti-neuropathic medications currently in use, such as some anti-depressants and anti-convulsants, have several drawbacks, specifically excessive sedation and drowsiness (5). Results of randomized controlled trials have yielded that patients persist to have, on average, a pain of moderate severity despite taking their prescribed pain medications (6).

A diversity of interventional options currently exists with variable efficacy, such as nerve block with local anesthetic and steroids, cryoablation, transcutaneous electrical nerve stimulation, surgical neurectomy, and orchidectomy (7).

The promising results of radiofrequency (RF) technology have attracted the attention of pain clinicians to use it in many chronic pain conditions (8). The usage of pulsed radiofrequency (PRF) in the treatment of chronic groin pain and orchialgia has been reported in limited case series (9-12).

The aim of this study is to evaluate the analgesic efficacy of PRF applied to the ilioinguinal nerve and the genital branch of the genitofemoral nerve for patients suffering from chronic post-surgical orchialgia.

\section{Methods}

This study was approved by the ethical committee of the faculty of medicine, and written informed consent was obtained from each patient. Seventy adult

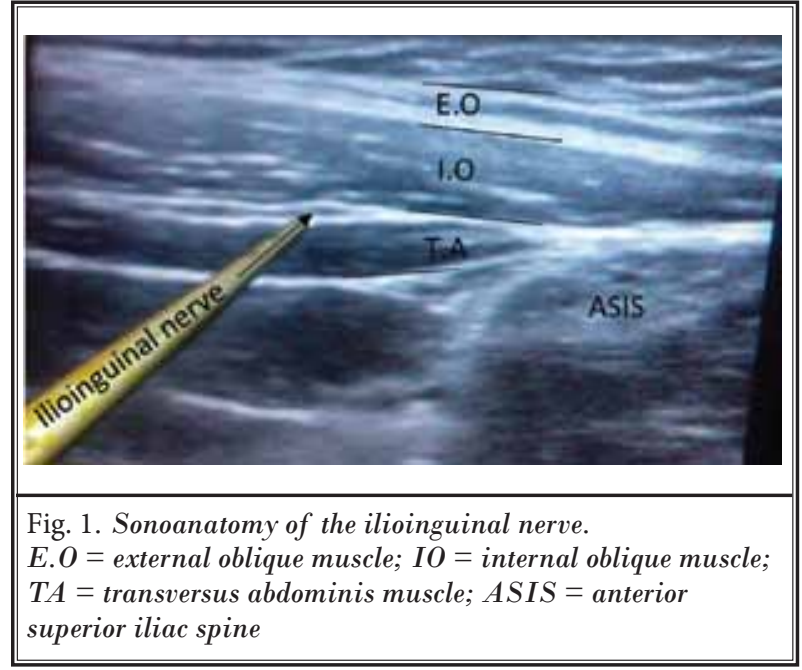

men complaining of chronic post-surgical orchialgia of at least 3 months that were referred from the andrology clinic to our interventional pain unit were consecutively enrolled. The trial was registered at www.anzctr. org.au, and the identification number for the registry is: ACTRN12616000649415.

The inclusion criteria were patients with chronic scrotal pain (orchialgia) that fulfilled the following criteria: 1) pain intensity $\geq 5$ on the visual analog scale (VAS), 2) pain that lasted for more than 3 months after groin surgeries, 3) failed conservative treatment with nonsteroidal anti-inflammatory drugs (NSAIDs), and 4) showed more than $50 \%$ reduction of their orchialgia on the VAS for at least 6 hours following spermatic cord block with $6 \mathrm{~mL}$ of lidocaine $2 \%$.

The exclusion criteria were: 1) patients with other causes of scrotal pain, e.g., epididymitis, orchitis, and hydrocele, 2) patients complaining of groin infection and coagulopathy, 3) patients suffering from hypertension, ischemic heart disease, or psychiatric disorders, and 4) patients allergic to study medications and concomitant use of NSAIDS or monoamine oxidase inhibitors.

The patients were randomly allocated to 2 groups by using a computer-created number list, which was concealed in sealed envelopes without blocking, and they were open by the researcher before application of the procedure.

The PRF group ( $n=35)$ was the interventional group, in which PRF was applied to the ilioinguinal nerve and the genital branch of the genitofemoral nerve using a NeuroTherm 2000 RF generator (NeuroTherm, Inc., Wilmington, MA). We sterilized the lower abdomen from the umbilicus to the scrotum, then, we started the ultrasound (US) scanning using an M-Turbo machine (SonoSite, Inc., Bothell, WA) with a superficial linear probe $10 \mathrm{HZ}$ frequency. The scanning process was implemented from the anterior superior iliac spine towards the umbilicus, keeping the shadow of iliac bone in view. The ilioinguinal nerve was identified in the fascial splitting area between the fascia transversalis and internal oblique muscles, just medial to the shadow of iliac bone (Fig. 1). A 22-gauge, $10 \mathrm{~cm}$ RF needle with a $10 \mathrm{~mm}$ active tip was introduced out of plane towards the nerve, and localization was further confirmed by producing a groin pain at $50 \mathrm{HZ}$ and less than 0.5 volt sensory stimulation and contraction of the lower outer quadrant of the abdominal muscles at 2 $\mathrm{HZ}$ and less than 1 volt motor stimulation. After satisfactory needle position was confirmed, PRF was initiated using the following parameters: voltage output: 
40-60 V; $2 \mathrm{~Hz}$ frequency; $20 \mathrm{~ms}$ pulses in a one-second cycle, 120 second duration per cycle and $42^{\circ} \mathrm{C}$ plateau temperature. PRF was applied for 4 cycles. The genital branch of the genitofemoral nerve was targeted under US guidance with the same probe, where the assistant held the testis and the cord and the probe was applied transversely just distal to the external inguinal ring; the deferent duct is visible as a non-compressible, internal hypoechoic structure with a hyperechoic horizon (Fig. 2). A 22-gauge, $10 \mathrm{~cm} \mathrm{RF}$ needle with a $10 \mathrm{~mm}$ active tip was advanced out of plane just lateral to the deferent duct and manipulated to achieve scrotal pain at $50 \mathrm{HZ}$ and less than 0.5 volt sensory stimulation. After localization of the nerve, PRF was commenced with the same parameters applied for the ilioinguinal nerve.

The sham group ( $n=35)$ was the control group, and the same procedure applied for the interventional group was done without giving PRF. The procedure was done by a senior staff pain clinician.

Two weeks following the procedure, the postoperative analgesic protocol was started and consisted of either duloxetine tablets initiated at a dose of $30 \mathrm{mg}$, increased up to $60 \mathrm{mg}$ according to the response as a first drug treatment, and/or pregabalin initiated at a dose of $50 \mathrm{mg}$, titrated according to the efficacy and side effects every week as a second drug treatment, and/or tramadol initiated at a dose of $50 \mathrm{mg}$ titrated according to efficacy and side effects as a third drug treatment. The postoperative follow-up (regarding pain assessment and treatment protocol) was accomplished by a pain clinician blinded to the study intervention.

The primary outcome variable was the percentage of patients that showed $>50 \%$ reduction of their VAS pain score (from baseline values), measured at 3 months post-procedure. The secondary outcome variables were the scrotal pain intensity measured by a VAS, the percentage of patients that did not require additional postoperative analgesics, the total analgesic consumption (duloxetine, pregabalin, or tramadol), and the global perceived effect (GPE) during the 3-month follow-up period. The GPE was assessed by a 7-point Likert-like verbal rating scale where: extremely dissatisfied $=1$, dissatisfied $=2$, somewhat dissatisfied $=3$, undecided $=$ 4 , somewhat satisfied $=5$, satisfied $=6$, and extremely satisfied $=7$.

\section{Statistical Analysis}

The statistical analysis was carried out on a personal computer using SPSS Version 22.0 (IBM Corpo-

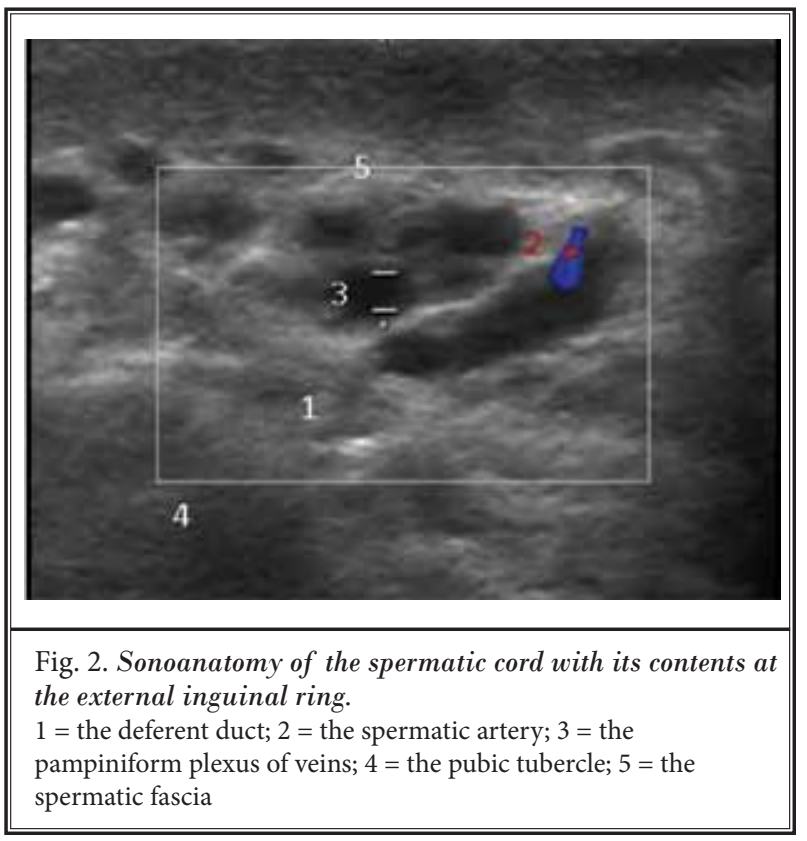

ration, Armonk, NY). Normality of continuous data distribution was tested with the Anderson-Darling test prior to further statistical analysis. Categorical data were described as number and percent, and comparisons were made by chi-square and Fisher's exact tests. Continuous data were described as mean \pm standard deviation (SD) or $95 \%$ confidence interval $(\mathrm{Cl})$, and point-by-point comparison was done by unpaired Student's t-test. A linear mixed-effects model for repeated measures was used for analysis of VAS pain scores over time $(2,4,6,8$, and 12 weeks postprocedural), examining the following effects: group, time, and group-by-time interaction, followed by post-hoc test with Beferroni correction for multiple comparisons. Medians and interquartile ranges were used for skewed data (GPE), and comparisons were made using the Mann-Whitney $U$ test. $P<0.05$ was considered statistically significant.

Based on a retrospective analysis of our institutional work, we found that $75 \%$ of patients treated with $\mathrm{PRF}$, in comparison to $33 \%$ of patients managed with medical treatment, showed more than $50 \%$ reduction of VAS pain score from baseline values. Using these assumptions, the estimated sample size for the 2-sample comparison of percentages revealed a minimum sample size of 28 patients in each study group would achieve a power of $80 \%$, assuming a type I error of 0.05 . We enrolled 35 patients in each group to allow for drop-outs. 


\section{Results}

Eighty patients were assessed for eligibility, 10 patients were excluded due to unsatisfactory response to spermatic cord block, 4 patients were lost to follow-up, 6 patients were excluded due to protocol violation, and

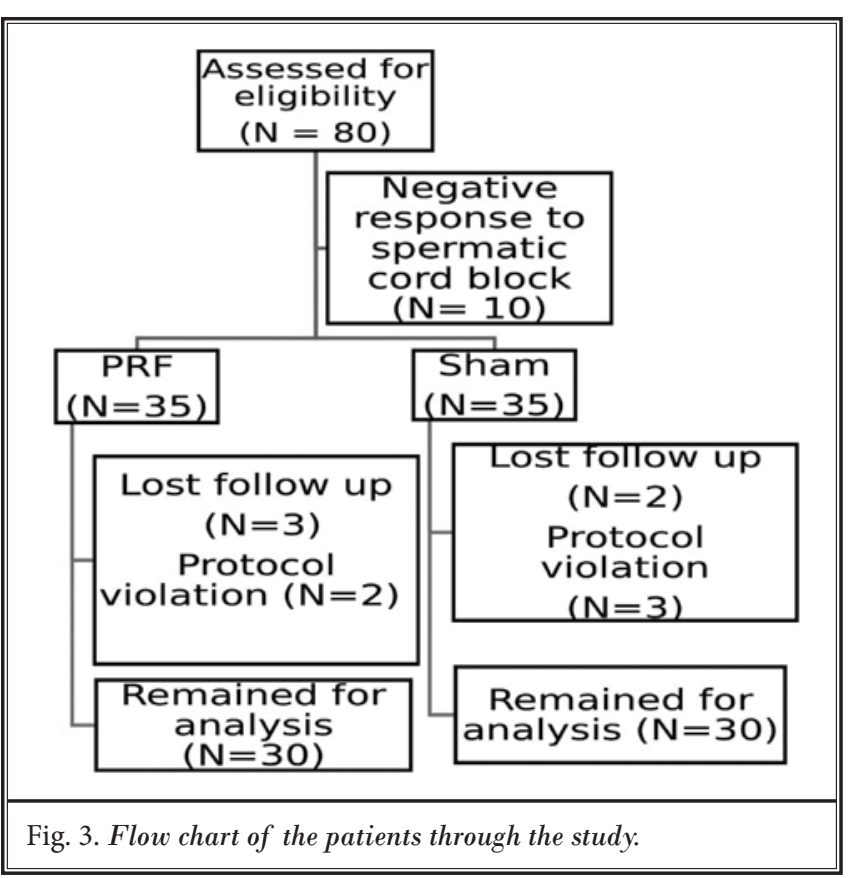

Table 1. Demographic data and patients' characteristics.

\begin{tabular}{||l|c|c|c|}
\hline \multirow{2}{*}{\multicolumn{1}{|c|}{ Variable }} & PRF & Sham & \multirow{2}{*}{ P-Value } \\
\cline { 2 - 4 } & $\mathbf{( n = 3 0 )}$ & $\mathbf{( n = 3 0 )}$ & \\
\hline Age $(\mathrm{yrs})$ & $35.8 \pm 9.7$ & $37.7 \pm 10.4$ & 0.467 \\
\hline BMI $\left(\mathrm{kg} / \mathrm{m}^{2}\right)$ & $28.3 \pm 3.4$ & $27.9 \pm 38$ & 0.935 \\
\hline $\begin{array}{l}\text { Type of Surgery } \\
\text { Varicocelectomy/Herniorrhaphy }\end{array}$ & $19 / 11$ & $18 / 12$ & 0.913 \\
\hline
\end{tabular}

Table 2. Postoperative analgesic requirements during 3 months follow-up.

\begin{tabular}{|l|c|c|}
\hline \multicolumn{1}{|c|}{ Variable } & $\begin{array}{c}\text { PRF } \\
(\mathbf{n}=\mathbf{3 0})\end{array}$ & $\begin{array}{c}\text { Sham } \\
(\mathbf{n = 3 0})\end{array}$ \\
\hline Patients with > 50\% Reduction of VAS, (n, \%) & $24(80 \%)$ & $7(23.33 \%)$ \\
\hline $\begin{array}{l}\text { Patients that did not Require Analgesics, } \\
\text { (n, \%) }\end{array}$ & $15(50 \%)$ & $1(3.3 \%)$ \\
\hline Duloxetine 30 mg & $10(33.3 \%)$ & $16(53.3 \%)$ \\
\hline Duloxetine 60 mg & $5(16.7 \%)$ & $9(30 \%)$ \\
\hline Duloxetine 60 mg + pregabalin & $0(0 \%)$ & $3(10 \%)$ \\
\hline Duloxetine 60 mg + pregabalin + tramadol & $0(0 \%)$ & $1(3.3 \%)$ \\
\hline
\end{tabular}

60 patients (30 in each group) remained for the analysis (Fig. 3).

There was not a statistically significant difference among the 3 groups with respect to demographic data and patient characteristics (Table 1).

The percentage of patients that showed $>50 \%$ reduction of their VAS pain score was $80 \%(24 / 30)$ in the PRF group, compared to $23.33 \%(7 / 30)$ in the sham group. The percentage of patients that did not require additional analgesic treatment was $50 \%(15 / 30)$ in the PRF group, compared to $3.3 \%$ $(1 / 30)$ in the sham group. The percentage of patients that required duloxetine at a dose of $30 \mathrm{mg}$ was $33.3 \%(10 / 30)$ in the PRF group, compared to $53.3 \%(16 / 30)$ in the sham group, and duloxetine at a dose of $60 \mathrm{mg}$ was required in $16.7 \%$ (5/30) of patients in the PRF group, compared to $30 \%$ (9/30) of patients in the sham group. Three patients in the sham group required duloxetine $60 \mathrm{mg}+$ pregabalin $100 \mathrm{mg}$ and one patient required duloxetine 60 mg + pregabalin 150 + tramadol 100 mg (Table 2).

The linear mixed model analysis of VAS pain scores revealed a significant reduction of the mean overall VAS pain score over time between the groups $(P<0.01)$, with a significant time and group-by-time interaction. Moreover, there was a significant reduction of the mean VAS score (between groups) at all of the measured time-points $(2,4,6,8$, and 12 weeks post-procedure) $(P=0.001)$ in the PRF group in comparison to the sham group (Table 3).

There was a significant improvement of the GPE in the PRF group in comparison to the sham group, evaluated after 6 and 12 weeks post-procedure, respectively (median, interquartile range) $[6(4: 7)]$ vs. $[4(1: 7)]$ and $[6(3: 7)]$ vs. $[4(2: 7)](P=0.00)$ (Fig. 4).

\section{Discussion}

In this randomized, double-blind, shamcontrolled, clinical trial, we found that PRF applied to the ilioinguinal nerve and the genital branch of the genitofemoral nerve for patients suffering from chronic postsurgical orchialgia significantly reduced pain intensity and decreased the demand for pain medications during a 3-month follow-up period.

In agreement with our results, Venkataparthasarathy and Ather (11), in their retrospective analysis of 15 cases of ilioinguinal and genitofemo- 
Table 3. Intensity of pain measured by a VAS.

\begin{tabular}{|c|c|c|c|}
\hline \multirow{2}{*}{ VAS } & PRF & Sham & \multirow{2}{*}{ P-Value } \\
\cline { 2 - 3 } & $(\mathbf{n}=\mathbf{3 0})$ & $\mathbf{( n = 3 0 )}$ & \\
\hline Pre-Procedure & $5.97(5.60-6.38)$ & $5.90(5.54-6.37)$ & 0.812 \\
\hline 2 wks & $2.07(1.61-2.58)$ & $4.63(4.12-5.19)$ & 0.001 \\
\hline 4 wks & $2.2(1.81-2.71)$ & $3.83(3.34-4.36$ & 0.001 \\
\hline 6 wks & $2.23(1.79-2.71)$ & $3.53(3.06-4.03)$ & 0.001 \\
\hline 8 wks & $2.40(1.92-2.89)$ & $3.17(2.8-3.57)$ & 0.016 \\
\hline 12 wks & $2.40(1.92-2.89)$ & $3.17(2.79-3.56)$ & 0.001 \\
\hline
\end{tabular}

Data are expressed as mean $(95 \% \mathrm{CI})$.

ral neuralgia treated with PRF on the appropriate nerve (ilioinguinal or genitofemoral), showed that the mean VAS pain score was reduced from 7.5 pre-procedure to 3.2 at 6 months post-procedure, and the number of patients that did not require additional post-procedure analgesic medications was 6. Moreover, Cohen and Foster (9) performed PRF on ilioinguinal or genitofemoral nerves for 3 patients complaining of groin pain or orchialgia, and they reported a complete post-procedure pain resolution during a 6-month follow-up period.

In contrast to the previously mentioned studies, the current study is unique in its design that is shamcontrolled, which is intrinsically difficult in chronic pain management. Furthermore, we included only the patients who showed more than $50 \%$ reduction of their VAS pain score in response to diagnostic spermatic cord block, to exclude patients with psychiatric problems, as well as secondary gain with malingering associated with chronic pelvic pain and to be sure that the pain signals came from the nerves in the cord and were not a referred pain from a mid-ureteral stone, inguinal hernia, aortic or common iliac artery aneurysms, or lower back disorders.

Several neurodestructive techniques, such as surgical rhizotomy, chemical neurolysis, and cryoablation, have been employed in the management of chronic pain states with variable efficacy (13). Cryoablation has been shown to provide effective, long-lasting pain relief when applied to peripheral nerves $(14,15)$. Unfortunately, the analgesic efficacy and duration of cryoablation depend basically on the extent of cold-induced axonal damage of neuronal tissue; thus, application of cryoablation to nerves such as the ilioinguinal and genitofemoral nerves that provide sensation to the genital region is unfavorable practice in interventional pain management.

The RF technology has gained wide spread use in interventional pain management. It has been conven-

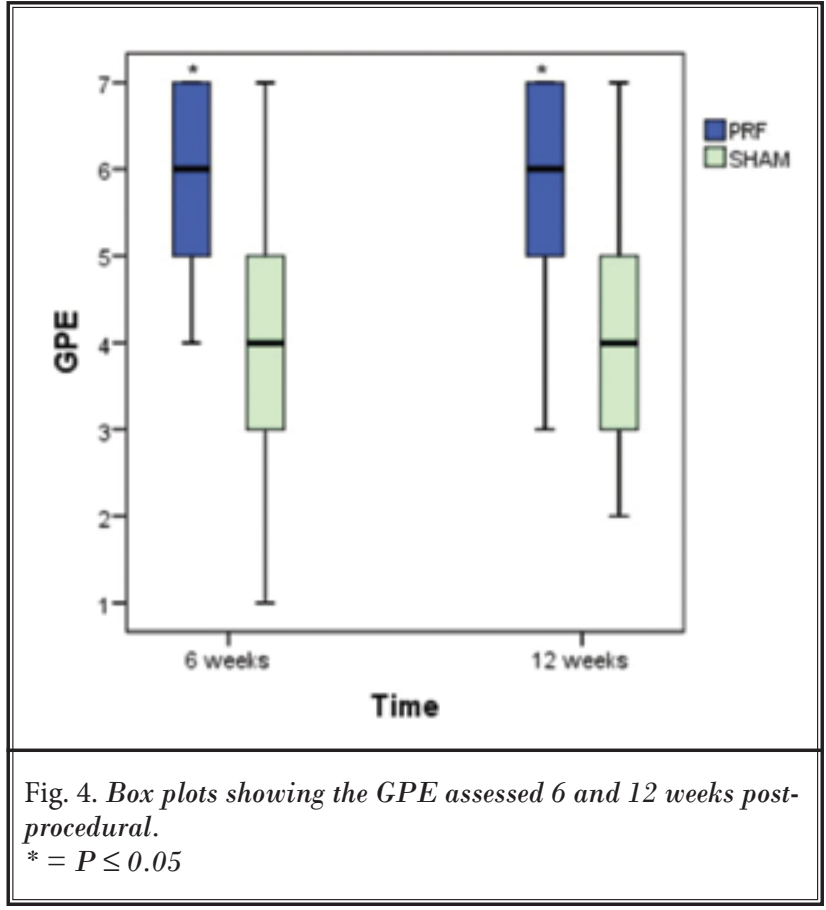

tionally used for interruption of pain impulses through heat-induced neurodestruction (16). Although conventional RF has a clear effective and long-standing pain relief, it carries the risks of dysthesia, sensory loss, and motor weakness. Such drawbacks make its application on peripheral nerves undesirable (17). In contrast, PRF is an alternative mode that interrupts the pain impulses through biological changes rather than neurodestruction (18).

To improve the antinociceptive effect of PRF, we applied it for an extended duration, 4 rounds of 120 seconds each rather than one round (120 seconds) that is repeatedly mentioned in previous studies (8). In this context, animals subjected to increased exposure time to PRF current from 2 to 6 minutes showed a significant antiallodynic effect without motor impairment (19). In our follow-up period, we did not report any neuropathic symptoms or signs related to the usage of PRF apart from some tingling sensations at the site of needle entry in a few patients that disappeared spontaneously within 4 or 5 days post-procedure.

Chronic post-surgical chronic scrotal pain or orchalgia is essentially due to unintentional injury or entrapment of the ilioinguinal and the genital branch of the genitofemoral nerve at the surgical site in their course at the groin region (Fig. 5), so interruption of pain impulses carried by these nerves by PRF was the main target in the current study. 


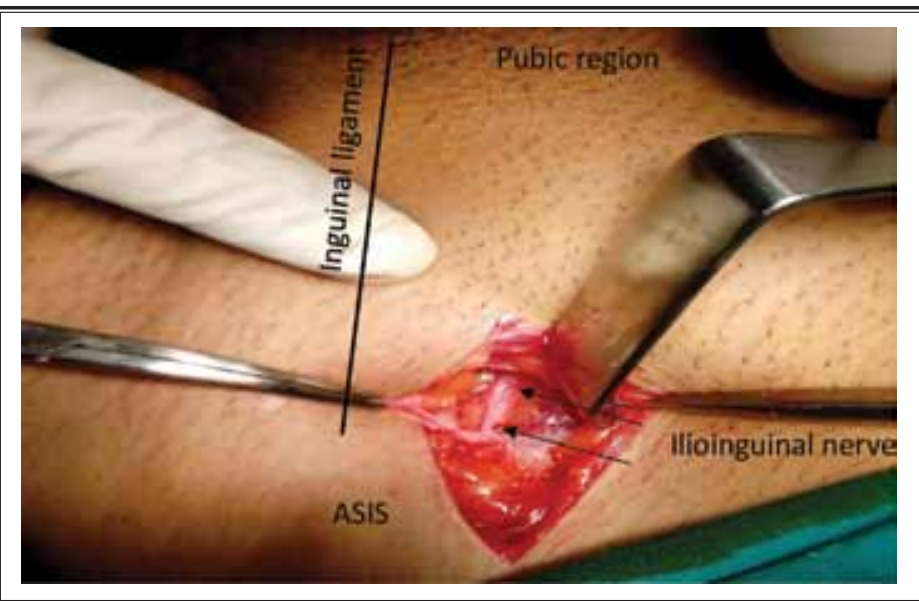

Fig. 5. An image of the surgical field of the varicocelectomy incision, demonstrating the course of the ilioinguinal nerve.

ASIS = anterior superior iliac spine

The arrows point to the course of the ilioinguinal nerve between the fascia transversalis and internal oblique muscle that could be injured during surgical dissection.

In this study, we clearly identified the ilioinguinal nerve under US guidance, just medial to the anterior superior iliac spine, in the fascial splitting area between internal oblique muscle and fascia transversalis, and further confirmation was achieved through nerve stimulation. In contrast, the genital branch of the genitofemoral nerve is a small nerve embedded in the fascial coverings of the spermatic cord (20) that could not be visualized by the US; alternatively, we placed the RF needle on the lateral region of the spermatic cord, which is a common course of the genital nerve, and advanced medially to pick it with the nerve stimulator.

In contrast to the mechanism by which corticotrophinreleasing factor controls pain, that is tissue thermocoagulation, the anti-allodynic mechanism of PRF is unclear, but it may involve a temperature-independent effect mediated by a rapidly changing electrical field (21). Sandkühler and colleagues (22) identified a robust long-term depression of synaptic transmission in substantia gelatinosa neurons that can be induced by a lowfrequency stimulation of the primary afferent A-delta fibers. In this context, Van Zundert et al (23) demonstrated an increase of c-fos expression in the dorsal horn cells after exposure of the cervical dorsal root ganglion to different RF modalities, which was not temperature dependent. Moreover, Hagiwara and associates (24) claimed that the analgesic action of PRF involves an enhancement of the noradrenergic and the serotonergic descending pain inhibitory pathways in an experimental pain model.

Regarding postoperative analgesic protocol, we assumed (from our anecdotal experience) that combination of multiple drugs is more beneficial than a maximum effective dose of each drug $(5,25)$. Therefore, we did not increase the dose of duloxetine above $60 \mathrm{mg}$ or pregabalin above $150 \mathrm{mg}$, and we preferred combination treatment to improve the patient's compliance and minimize side effects, which is consistent with the general concept of management of chronic neuropathic pain $(6,26)$.

We cannot deny that duloxetine was able to reduce the pain intensity significantly by time, but the GPE was significantly better in the PRF group. Duloxetine is a serotoninnorepinephrine reuptake inhibitor; its exact analgesic action in human is unknown. It is suggested that duloxetine increases the level of serotonin and norepinephrine in synaptic clefts at the spinal and the supra-spinal levels, activating the descending pain inhibitory system (27).

This study was limited by the follow-up period, which was only 3 months.

\section{Conclusions}

For patients suffering from chronic post-surgical orchialgia, PRF applied to the ilioinguinal nerve and the genital branch of the genitofemoral nerve is an effective treatment modality. It provides long-lasting pain relief and decreases the demand for pain medications. 


\section{References}

1. Aasvang E, Kehlet H. Chronic postoperative pain: The case of inguinal herniorrhaphy. Br J Anaesth 2005; 95:69-76.

2. Alfieri S, Amid PK, Campanelli G, Izard G, Kehlet H, Wijsmuller AR, Di Miceli $D$, Doglietto GB. International guidelines for prevention and management of post-operative chronic pain following inguinal hernia surgery. Hernia 2011; 15:239-249.

3. Tojuola B, Layman J, Kartal I, Gudelogul A, Brahmbhatt J, Parekattil S. Chronic orchialgia: Review of treatments old and new. Indian J Urol 2016; 32:21-26.

4. Macrae WA. Chronic post-surgical pain: 10 years on. $\mathrm{Br}$ J Anaesth 2008; 101: 77-86.

5. Dworkin RH, O'Connor AB, Backonja $M$, Farrar JT, Finnerup NB, Jensen TS, Kalso EA, Loeser JD, Miaskowski C, Nurmikko TJ, Portenoy RK, Rice AS, Stacey BR, Treede RD, Turk DC, Wallace MS. Pharmacologic management of neuropathic pain: Evidence-based recommendations. Pain 2007; 132:237-251.

6. Dworkin RH, O'Connor AB, Audette J, Baron R, Gourlay GK, Haanpää ML, Kent JL, Krane EJ, LeBel AA, Levy RM, Mackey SC, Mayer J, Miaskowski C, Raja SN, Rice AS, Schmader KE, Stacey B, Stanos S, Treede RD, Turk DC, Walco GA, Wells $C D$. Recommendations for the pharmacological management of neuropathic pain: An overview and literature update. Mayo Clin Proc 2010; 85:S3-S14.

7. Davis BE, Noble MJ, Weigel JW, Foret JD, Mebust WK. Analysis and management of chronic testicular pain. J Urol 1990; 143:936-939.

8. Shi Y, Wu W. Treatment of neuropathic pain using pulsed radiofrequency: A meta-analysis. Pain Physician 2016; 19:429-444.

9. Cohen SP, Foster A. Pulsed radiofrequency as a treatment for groin pain and orchialgia. Urology 2003; 61:645.

10. Rozen D, Parvez U. Pulsed radiofrequency of lumbar nerve roots for treatment of chronic inguinal herniorraphy pain. Pain Physician 2006; 9:153-156.

11. Venkataparthasarathy R, Ather MH. IIioinguinal and genitofemoral neuralgia: Pulsed radiofrequency. A novel technique. Eur J Pain 2006; 10:S1.

12. Rozen D, Ahn J. Pulsed radiofrequency for the treatment of ilioinguinal neuralgia after inguinal herniorrhaphy. Mt Sinai J Med 2006; 73:716-718.

13. Nadler SF, Weingand K, Kruse RJ. The physiologic basis and clinical applications of cryotherapy and thermotherapy for the pain practitioner. Pain Physician 2004; 7:395-399.

14. Kim CH, Hu W, Gao J, Dragan K, Whealton $\mathrm{T}$, Julian C. Cryoablation for the treatment of occipital neuralgia. Pain Physician 2015; 18: $\mathrm{E}_{3} 63-\mathrm{E}_{3} 68$.

15. Byas-Smith MG, Gulati A. Ultrasoundguided intercostal nerve cryoablation. Anesth Analg 2006; 103:1033-1035.

16. Van Zundert J, Raj P, Erdine S, van Kleef $M$. Application of radiofrequency treatment in practical pain management: State of the art. Pain Pract 2002; 2: 269-278.

17. Vatansever D, Tekin I, Tuglu I, Erbuyun K, Ok G. A comparison of the neuroablative effects of conventional and pulsed radiofrequency techniques. Clin J Pain 2008; 24:717-724.

18. Erdine S, Bilir A, Cosman ER, Cosman ER Jr. Ultrastructural changes in axons following exposure to pulsed radiofrequency fields. Pain Pract 2009; 9:407-417.

19. Erdine S, Yucel A, Cimen A, Aydin S, Sav A, Bilir A. Effects of pulsed versus conventional radiofrequency current on rabbit dorsal root ganglion morphology. Eur J Pain 2005; 9:251-256.
20. Standring S. Gray's Anatomy. 39th ed. Elsevier Churchill Livingstone, Edinburgh, 2005, pp 1125.

21. Tanaka N, Yamaga M, Tateyama S, Uno T, Tsuneyoshi I, Takasaki M. The effect of pulsed radiofrequency current on mechanical allodynia induced with resinifera toxin in rats. Anesth Analg 2010; 111:784-790.

22. Sandkühler J, Chen JG, Cheng G, Randi M: Low-frequency stimulation of afferent Adelta-fibers induces long-term depression at primary afferent synapses with substantia gelatinosa neurons in the rat. J Neurosci 1997; 17:6483-6491.

23. Van Zundert J, de Louw AJ, Joosten EA, Kessels AG, Honig W, Dederen PJ, Veening JG, Vles JS, van Kleef M. Pulsed and continuous radiofrequency current adjacent to the cervical dorsal root ganglion of the rat induces late cellular activity in the dorsal horn. Anesthesiology 2005; 102:125-131.

24. Hagiwara S, Iwasaka H, Takeshima N, Noguchi T. Mechanisms of analgesic action of pulsed radiofrequency on adjuvant-induced pain in the rat: Roles of descending adrenergic and serotonergic systems. Eur J Pain 2009; 13:249-252.

25. Finnerup NB, Sindrup SH, Jensen TS. The evidence for pharmacological treatment of neuropathic pain. Pain 2010; 150:573-581.

26. Gilron I, Bailey JM, Tu D, Holden RR, Jackson AC, Houlden RL. Nortriptyline and gabapentin, alone and in combination for neuropathic pain: A doubleblind, randomised controlled crossover trial. Lancet 2009; 374:12521261.

27. Jones CK, Peters SC, Shannon HE. Efficacy of duloxetine, a potent and balanced serotonergic and noradrenergic reuptake inhibitor, in inflammatory and acute pain models in rodents. J Pharmacol Exp Ther 2005; 312:726-732. 
